\title{
The Amen Anti-Addiction Intervention: Theoretical Foundations of a Novel Neurotrophic Protocol for the Prevention and Treatment of Addiction
}

\author{
Nun Sava-Siva Amen-Ra \\ The Amen Institute of Ascetic Psychology, 25101 Chimney House Court, Damascus, USA \\ Email: Amen-Ra@AmentaEliteAthlete.com
}

Received 22 January 2014; revised 21 February 2014; accepted 20 March 2014

Copyright (c) 2014 by author and Scientific Research Publishing Inc.

This work is licensed under the Creative Commons Attribution International License (CC BY). http://creativecommons.org/licenses/by/4.0/

\section{Abstract}

The Amen Anti-Addiction Intervention (AAI) is an austere regimen intended to eradicate addictive impulses and behaviors. The theoretical and empirical foundations of this intervention are explicated herein. The AAI entails daily cyclic fasting, chronic caloric restriction, supplementation with specific neutraceutical agents, rigorous exercise, and minimalist meditation. The physiological focus of the AAI is twofold: the augmentation of neurogenesis in a manner analogous to the effect of psychotropic drugs and the activation of areas in the brain involved in the regulation of emotional arousal and reward. The psychological focus of the AAI is similarly twofold: the attenuation of anxiety and/or impulsivity and the cultivation of mental mastery and/or self-control. It shall be argued that the AAI, by virtue of its explicit integration of biological, psychological, and sociological elements-each of which evidently influences addiction-will plausibly be an improvement over existing interventions aimed at the treatment of addiction.

\section{Keywords}

Neurogenesis, Addiction, Neurotrophins, Fasting, Caloric Restriction, Substance Abuse

\section{Introduction}

There is evidence extant in extensive scientific literature that addiction to various agents and behaviors shares common molecular mechanisms. Further, there is considerable scientific support for the supposition that biological, psychological, and sociological factors influence the propensity of individuals to succumb to addiction [1]. 
As such, it would seem that the most effective interventions should emanate from the integration of these three domains - biology, psychology, and sociology. In short, the most prudent theoretical perspective for the prevention and treatment of addiction is ideally bio-psycho-social in its overall orientation. Such is the case with the protocol proposed herein. The Amen Anti-Addiction Intervention (AAI) is an outgrowth of the Amen Optimal Health Protocol (AOHP). The aim of the AOHP is to incorporate into a single practical system every intervention that has proven to extend maximum longevity and/or optimize health in model organisms [2] [3]. In accordance with scientific data amassed over nearly a century, the AOHP entails the following physiological elements: cyclic fasting amounting to $23+$ hours per day; dietary restriction amounting to $25 \%$ - $50 \%$ reductions in customary caloric intake consumed in a single span of an hour or less; a fixed, virtually invariant, schedule of feeding consonant with the solar day; vigorous daily exercise; and supplementation with select nutrients and neutraceuticals (i.e. natural compounds with defined bioactive agents). The AOHP entails a single psychological element: minimalist meditation aimed at attenuating anxiety and instilling a state of psychic equanimity. Lastly, the AOHP entails a sociological element—specifically, social support conferred by the small community of committed practitioners of the analogous Amen protocols.

\section{Exposition of the Amen Anti-Addiction Intervention}

In the course of exploring the biological bases of drug addiction, it became clear to the Author that the same neurological systems involved in drug addiction are also altered by intermittent fasting, caloric restriction, and scheduled feeding. Additionally, the Author found evidence for the efficacy of exercise in the excitation of reward systems in the brain - systems acted upon by various drugs of abuse. The Author apprised himself of evidence that impulsivity and anxiety influence the propensity for addiction. This revealed the relevance of meditation as a means of attenuating impulsivity and anxiety, thereby supporting its suitability in the prevention and mitigation of addiction. Social support is crucial in the adoption of an intervention as rigorous as the AOHP. Adherents invariably find the support provided by fellow practitioners to be particularly valuable. Given the evidence that social environs influence addictive behaviors, it is plausible that the social support extant in the community of AOHP/AAI adherents could counterbalance the deleterious social signals which promote and sustain illicit substance use. This realization prompted the Author to consider other elements of the AOHP that are potentially conducive to the attenuation of addiction.

Arguments have been advanced that much substance abuse is intended to "self-medicate" symptoms of mental disorders, particularly depression and anxiety [1]. According to this line of thought, drug abuse is driven by the desire to modulate mood, particularly depressive or anxious states. Thus, illicit drugs are "intended" to exert the same effect as licit psychotropic drugs - that is, to elevate mood. The molecular mode of action of common classes of psychotropic drugs used to treat depression and anxiety is understood to a considerable extent. One such molecular modality is the induction of endogenous neurotrophic factors in the brain. Neurotrophic factors include peptides or proteins such as brain-derived nerve growth factor (BDNF), which promote the growth and development of neurons in the brain. Diverse classes of psychotropic drugs including non-selective antidepressants (e.g. imipramine), norepinephrine-selective reuptake inhibitors (e.g. desipramine) and serotonin-selective reuptake inhibitors (e.g. fluoxetine) increase the generation of new neurons in the brain-that is, they induce neurogenesis [4]. Thus, neurogenesis is thought to mediate the mood elevation that commonly accompanies pharmacotherapeutic treatment of mood disorders. An intervention that is able to induce increased neurogenesis in relevant regions of the brain known to modulate mood could likely exert an effect—preventative or therapeutic —on illicit drug use and other addictive behaviors potentiated by affective impairments. Caloric restriction is such an intervention. It has been demonstrated that cyclic fasting and/or chronic caloric restriction (CF/CR) increase the expression of BDNF, resulting in enhanced generation of neurons in such regions as the hippocampus, neocortex, and striatum of experimental mammals [5]. Conversely, it has been shown that several drugs of abuse impair the proliferation of new neurons in the brain, particularly in the hippocampus. Such drugs include alcohol [6], opiates [7], and stimulants [8] [9]. We herein hypothesize that CF/CR can conceivably counter addiction by attenuating impaired neurogenesis engendered by several classes of commonly abused drugs.

Complementing the evidence that fasting and caloric restriction may improve psychological well-being by mimicking the neurogenic effects of psychotropic drugs is evidence that exercise acts in a similar manner. This effect was established by Huang and colleagues [10], who found that daily voluntary exercise in experimental mice was as effective as the antidepressant drug fluoxetine in inducing an antidepressant effect. The molecular mechanisms underlying this effect in both the fluoxetine and exercise intervention groups was found to be an 
induction of neurogenesis and increased dendritic spine density in hippocampal neurons. Other empirical investigations have substantiated this experimental finding [11].

Stress is known to induce neuronal loss in specific regions of the adult brain [12]. Interventions that successfully alleviate stress have the potential to antagonize addiction by inducing an antidepressant, anxiolytic (i.e. anxiety reducing) effect. Meditation is one such intervention that has been shown to diminish depression, stress, and anxiety [13]. Meditation, moreover, has been applied as an intervention in the treatment of drug addiction. Witkiewitz [14] and colleagues found that a type of meditative technique termed Mindfulness-Based Relapse Prevention (MBRP) was able to reduce drug cravings and substance use in an efficacy trial. A randomized, controlled pilot study by Brewer [15] and colleagues found meditation to be as effective as cognitive behavioral therapy (CBT) in reducing stress among groups of clients undergoing substance abuse treatment. It has been found, moreover, that meditation of the MBRP variety increased unilateral hippocampal thickness in a group of practitioners as established by magnetic resonance imaging [16].

Certain natural substances are known to promote neurogenesis in particular regions of the brain, such as the hippocampus. Among such agents is the polar compound choline. Glenn [17] and colleagues demonstrated that administration of choline to adult rodents augmented expression of BDNF and consequently induced increased neurogenesis in the hippocampus. Administering this agent to developing rats exerted antidepressant like effects therein. Several studies have demonstrated the capacity of ginseng (Panax species) to induce neurogenesis in the hippocampus via induction of neurotrophic factors. A study by Jiang [18] and colleagues found that a chemical constituent of ginseng (Rg1) augmented neurogenesis in the hippocampal regions of mice via BDNF induction. The effect attenuated various indices of depression and stress in the experimental subjects, an effect that was comparable to the antidepressant imipramine, which the investigators employed as a positive control. Constituents of coffee (inclusive of caffeine) have equivocally been found to exert a protective effect on neurological function and this has been attributed to increases in hippocampal neurogenesis [19]. These and other non-toxic neutraceuticals are common constituents of the Amen Apothecary. Though the Amen Apothecary was originally formulated to advance the aim of lifespan extension, several of the substances contained in the formulary are known to be neurogenic. In addition to those already indicated, blueberry extract [20], cocoa [21], grape seed extract [22] the unicellular autotroph spirulina [23] garlic [24] soy [25], and conventional tea (Camellia senensis) [26], have each been found to induce neurogenesis. Indeed, it has been determined that diets rich in plant polyphenols may generally promote hippocampal neurogenesis [27]. The Amen Diet as such is substantially soybased, containing significant amounts of spirulina and garlic and therefore theoretically capable of heightening the neurogenic effect of the supplements constituting the Amen Apothecary proper. Importantly, the Amen Diet is thoroughly vegetal, containing no meat products. There is complementary evidence that dietary cholesterol compromises hippocampal neurogenesis [28] and, conversely, that cholesterol-lowering drugs (i.e. statins) promote neurogenesis in a manner analogous to antidepressants [29]. Given that animal products are the sole sources of naturally occurring cholesterol, a diet devoid of meat accommodates heightened hippocampal neurogenesis relative to omnivorous diets inclusive of meat.

It has been found that extracts of the South African herb, Aspalathus linearis (also known as Rooibos or red tea) inhibits the age related reduction in hippocampal thickness [30], an effect which may plausibly be related to enhanced neurogenesis. Tea—both traditional tea and red tea-is imbibed throughout the lengthy Amen Fast and this practice may further promote neurogenesis, potentially providing a protracted state of neurotrophic stimulation during the diurnal period.

It is evident that each element of the AAI plausibly possesses the potential to attenuate addictive behavior via an empirically established ability to induce regionally specific neurogenesis in a manner similar to psychotropic drugs. Six separate components serve this salutary end-cyclic fasting, caloric restriction, exclusively vegetal nutriment, exercise, neutraceuticals, and meditation. The multifaceted nature of the AAI, combined with its manifest molecular mimicry of the psychotropic medication modality and its absence of artificiality (and presumably deleterious pharmacologic side-effects) may make it inherently more effective than alternative preventative/ therapeutic interventions.

Though there is no current consensus on the etiology of drug addiction, there is considerable support for the role of molecularly mediated motivation [31]. More specifically, drugs of abuse are thought to alter the actions of neurotransmitters in particular regions of the brain associated with reward and motivation [32]. As explained in a review by McCarthy [33] and colleagues, dopamine serves a central role in regulating reward:

A major CNS dopaminergic pathway is the mesolimbic pathway. In this pathway, dopamine synthesized in the 
ventral tegmental area (VTA) of the mid brain is transported to the nucleus accumbens (NAc), amygdala, hippocampus, and prefrontal cortex (PFC) in the forebrain. The mesolimbic dopaminergic pathway is also referred to as the "reward pathway" because of its critical involvement in mediating rewarding effects of drugs such as cocaine (p. 439).

Additionally, impulsivity and impaired self-control are common, compelling, explanatory elements of theories and models of addictive behaviors [34]-[36]. According to such theories, addiction entails an inability to control impulses impelling engagement in drug use and the consistent failure to control such impulses ultimately eventuates in the compulsion of chronic drug use. If, indeed, excessive impulsivity potentiates drug abuse and addiction, it would seem that an intervention aimed extinguishing addiction should endeavor to attenuate impulsivity in order to enable the acquisition of self-control. Such is the case with the AAI.

It is herein hypothesized that cyclic fasting/caloric restriction (CF/CR) may be effective in the treatment of addiction insofar as it is operative in the two dimensions discussed above- that is, CF/CR may modulate molecularly mediated motivation and it may plausibly inhibit impulsivity. Evidence indicates that feeding restriction alters the chemistry of the brain such that pleasure centers (principally limbic structures) are activated [37]. This activation apparently enhances the pleasurability of hedonic stimuli such as food and illicit drugs. Fang [38] and colleagues found that electrical stimulation of specific limbic nuclei in recovering heroin addicts induced a state of euphoria comparable to the drug. Dopaminergic (dopamine sensitive) neurons play a prominent part in the perception of pleasure; heightened dopamine signaling accompanies the ingestion of food (especially after an extended interval of fasting) as well as certain psychoactive drugs [39]. Hypothetically, an extended (23-hr) period of food deprivation followed by a brief (1-hr) period of feeding should partially mimic the effect of drug administration on the dopaminergic system. This hypothesis is substantiated by an investigation by Yamamoto [37] and colleagues which explored the expression of genes in reward-related regions of the brain (prefrontal cortex, amygdala, and hypothalamus) in rodents subjected to fasting and restricted feeding. Analyses indicated that the most substantial alteration of expression was an upregulation of dopamine receptor signaling indicative of increased dopaminergic activity. The Author speculates that the similarity between the chemical cascade catalyzed by temporal food restriction and that induced upon drug ingestion may enable one to substitute the reward engendered by feeding for the reward which ordinarily accompanies intoxication. In the analogous context of alcohol addiction and exercise, Ozburn [40] has termed this concept "hedonic substitution".

Fasting entails self-imposed control over the impulse to eat. Foregoing feeding for 23 hours daily and partaking of a single evening meal of moderate energy density facilitates (and indeed constitutes) self-control. Appetitive impulses are suppressed during extended fasting and such suppression serves to strengthen one's psyche thereby enabling the attenuation of addiction according to the hypothesis advanced herein. The feasibility of fasting for 23 hours daily is experientially established by the Author's personal practice of the protocol for a period approaching a decade and his assistance in aiding patients in the successful adoption of the AOHP in the context of his private practice. The feasibility of fasting consistently for spans of 24+ hours has been empirically established by several clinical trials. In a review of caloric restriction studies in humans, Varady [41] compared the reported efficacy of daily caloric restriction versus intermittent fasting (feeding on alternating days for 24 hours). Varady identified 11 conventional caloric restriction trials (entailing 15\% - 60\% CR), 5 published intermittent fasting trials, and 2 theretofore unpublished trials conducted in his laboratory. Such studies (which ranged in duration from 4 - 24 weeks) suggest that consistent CR and fasting can be well tolerated for moderate periods whereas the Author's experience illustrates the tolerability of combined CR and daily fasting for an extensive, decade-long period without noticeable negative effects and considerable perceived positive effects (e.g. increased mental and physical energy, maintenance of ideal body weight and body composition, etc.).

Anxiety is among the effects of drug withdrawal [42]. Attenuating the anxiety associated with drug withdrawal may increase the odds that addicts undergoing treatment will abstain from drug abuse. Meditation has demonstrated some efficacy in the attenuation of anxiety [43]. The inclusion of meditative techniques in the AAI may thereby aid in drug rehabilitation via anxiety reduction. Further, meditation may mitigate impulsivity, which (as discussed above) is implicated in addiction. The Author [44] has developed and practiced a form of meditation that may be particularly effective at attenuating the impulsivity allegedly underlying addictive behaviors. In contrast to such meditative techniques as Mindfulness (the anti-addiction efficacy of which has indeed been established in short-term follow-up of 2 - 4 months) [45], Amen Meditation (AM) is "contentless", involving no imagery, verbal guidance or prolonged facilitation. These factors-namely, no necessity for facilitation after inconsiderable initial instruction—may make AM more effective insofar as it can be initiated more rapidly 
and executed independently by the practitioner/client. Briefly, AM entails an attempt to expurgate ideas, images, and emotions from the mind and suppress awareness of sensation in the body-it is an intentional inhibition of the impulse to think, feel, and move, an intentional imposition of insipidity or ataraxy. Amen meditation adopts the Jainist posture of kayotsarga (connoting in Prakrit "forgetting the body" [46]) or the Yogic posture of savasana (denoting in Sanskrit "corpse posture"). Kayotsarga is a relaxed, standing, statuesque posture. Alternatively, AM practitioners may adopt an immobile recumbent, horizontal posture-such is savasana. Merely maintaining such postures (for 10 - 20 minute intervals) requires impulse control—control of the urge to ambulate. Can the conscious control of thought and posture that AM entails be applied to addictive behavior; is it generalizable? Such is what I hypothesize- that AM is able to eliminate or attenuate chemical and behavioral addiction owing to its antagonism of impulsivity.

\section{Future Research}

Preliminarily, the Author proposes to conduct a pilot study, the intent of which is to ascertain the effect of the AAI on the treatment of addiction among individuals in a drug treatment facility. It is hypothesized that the AAI will prove more effective in inducing abstinence than the standard treatment entailing psychological interventions (e.g. cognitive behavioral-based therapy (CBT)) solely or with the aid of prescribed pharmaceutical drugs (e.g. opioid antagonists). The Author endeavors to execute the investigation in an outpatient addiction clinic over a 3-month period. Participants will be recruited from incoming clients seeking treatment for alcohol and drug addiction. Self-selected individuals will be incorporated into an alternative treatment group. The alternative treatment group shall be instructed in the implementation of the AAI, asked to record their food intake, be provided with select neutraceutical agents included in the Amen Apothecary (specifically the two types of teatraditional and red), asked to indicate the time and duration of fasting and exercise, and record the frequency and duration of meditation. The alternative treatment group shall be compared to a standard treatment group consisting of incoming clients assigned to conventional treatment-specifically, weekly individual and group psychotherapy sessions employing CBT as the primary treatment technique. The main outcome of interest (the dependent variable) shall be abstinence. Abstinence shall be defined as an absence of detectable drugs in random urinalyses over the study interval. The minor outcome of interest shall be a reduction in the intensity of addiction as assessed by an empirical index whose validity and reliability have been observationally established. The proportion of participants from the alternative and conventional groups who maintain drug abstinence (after the first month of the study's commencement) shall be compared to ascertain the existence of a statistically significant difference. In addition, differences in the intensity of addiction attested by the two groups shall be quantified and compared. Subsequent studies shall be aimed at integrating all elements of the AAI over increasingly lengthy intervals of investigation.

\section{References}

[1] Abadinsky, H. (2010) Drug Use \& Abuse: A Comprehensive Introduction. 7th Edition, Cenage Learning, Belmont.

[2] Amen-Ra, N. (2013) The Amen Optimal Health Protocol: An Exposition on the Experimental, Theoretical \& Practical Underpinnings of Lifespan Extension \& Optimal Health Promotion, a Discourse on the Method of Gerostasis, Volumes I-III. Amenta Press, Damascus.

[3] Amen-Ra, N. (2007) The Optimal Human Diet: Theoretical Foundations of the Amen Protocol. In: Robitaille, F.P., Ed., Diet Therapy Research Trends, Nova Science, New York, 177-240.

[4] Pechnick, R.N., Zonis, S., Wawrowsky, K., Cosgayon, R., et al. (2011) Antidepressants Stimulate Hippocampal Neurogenesis by Inhibiting p21 Expression in the Subgranular Zone of the Hippocampus. PLoS One, 6, e27290.

[5] Duan, W., Guo, Z. and Mattson, M.P. (2001) Brain-Derived Neurotrophic Factor Mediates an Excitoprotective Effect of Dietary Restriction in Mice. Journal of Neurochemistry, 76, 619-626. http://dx.doi.org/10.1046/j.1471-4159.2001.00071.x

[6] Herrera, D.G., Yague, A.G., Johnsen-Soriano, S., et al. (2003) Selective Impairment of Hippocampal Neurogenesis by Chronic Alcoholism: Protective Effects of an Antioxidant. Proceedings of the National Academy of Sciences of the United States of America, 100, 7919-7924. http://dx.doi.org/10.1073/pnas.1230907100

[7] Eisch, A.J. and Harburg, G.C. (2006) Opiates, Psychostimulants, and Adult Hippocampal Neurogenesis: Insights for Addiction and Stem Cell Biology. Hippocampus, 16, 271-286. http://dx.doi.org/10.1002/hipo.20161

[8] Sudai, E., Croitoru, O., Shaldubina, A., et al. (2011) High Cocaine Dosage Decreases Neurogenesis in the Hippocampus 
and Impairs Working Memory. Addiction Biology, 16, 251-260. http://dx.doi.org/10.1111/j.1369-1600.2010.00241.x

[9] Teuchert-Noodt, G., Dawirs, R.R. and Hildebrandt, K. (2000) Adult Treatment with Methamphetamine Transiently Decreases Dentate Granule Cell Proliferation in the Gerbil Hippocampus. Journal of Neural Transmission, 107, 133143. http://dx.doi.org/10.1007/s007020050012

[10] Huang, G.J., Ben-David, E., Tort Piella, A., et al. (2012) Neurogenomic Evidence for a Shared Mechanism of the Antidepressant Effects of Exercise and Chronic Fluoxitine in Mice. PLoS One, 7, e35901.

http://dx.doi.org/10.1371/journal.pone.0035901

[11] Curlik, D.M. and Shors, T.J. (2013) Training Your Brain: Do Mental and Physical (MAP) Training Enhance Cognition through the Process of Neurogenesis in the Hippocampus? Neuropharmacology, 64, 506-514. http://dx.doi.org/10.1016/j.neuropharm.2012.07.027

[12] Esch, T., Stefano, G.B., Fricchione, G.L. and Benson, H. (2002) The Role of Stress in Neurodegenerative Diseases and Mental Disorders. Neuroendocrinology Letters, 23, 199-208.

[13] Marchand, W.R. (2013) Mindfulness Meditation Practices as Adjunctive Treatments for Psychiatric Disorders. Psychiatric Clinics of North America, 36, 141-152. http://dx.doi.org/10.1016/j.psc.2013.01.002

[14] Witkiewitz, K., Lustyk, M.K. and Bowen, S. (2013) Retraining the Addicted Brain: A Review of Hypothesized Neurobiological Mechanisms of Mindfulness-Based Relapse Prevention. Psychology of Addictive Behaviors, 27, 351-365.

[15] Brewer, J.A., Sinha, R., Chen, J.A., et al. (2009) Mindfulness Training and Stress Reactivity in Substance Abuse: Results from a Randomized, Controlled Stage I Pilot Study. Substance Abuse, 30, 306-317. http://dx.doi.org/10.1080/08897070903250241

[16] Holzel, B.K., Carmody, J., Vangel, M., et al. (2011) Mindfulness Practice Leads to Increases in Regional Brain Gray Matter Density. Psychiatry Research: Neuroimaging, 191, 36-43. http://dx.doi.org/10.1016/j.pscychresns.2010.08.006

[17] Glenn, M.J., Adams, R.S. and McClurg, L. (2012) Supplemental Dietary Choline during Development Exerts Antidepressant-Like Effects in Adult Female Rats. Brain Research, 1443, 52-63. http://dx.doi.org/10.1016/j.brainres.2012.01.018

[18] Jiang, B., Xiong, Z., Yang, J., et al. (2012) Antidepressant-Like Effects of Ginsenoside Rg1 Are Due to Activation of the BDNF Signalling Pathway and Neurogenesis in the Hippocampus. British Journal of Pharmacology, 166, 18721887. http://dx.doi.org/10.1111/j.1476-5381.2012.01902.x

[19] Costa, M.S., Botton, P.H., Mioranzza, S., et al. (2008) Caffeine Improves Adult Mice Performance in the Object Recognition Task and Increases BDNF and TrkB Independent on phospho-CREB Immunocontent in the Hippocampus. Neurochemistry International, 53, 89-94. http://dx.doi.org/10.1016/j.neuint.2008.06.006

[20] Casadesus, G., Shukitt-Hale, B., Stellwagen, H.M., et al. (2004) Modulation of Hippocampal Plasticity and Cognitive Behavior by Short-Term Blueberry Supplementation in Aged Rats. Nutritional Neuroscience, 7, 309-316.

[21] Fernández-Fernández, L., Comes, G., Bolea, I., et al. (2012) LMN Diet, Rich in Polyphenols and Polyunstaturated Fatty Acids, Improves Mouse Cognitive Decline Associated with Aging and Alzheimer's Disease. Behavioural Brain Research, 228, 261-271. http://dx.doi.org/10.1016/j.bbr.2011.11.014

[22] Yoo, D.Y., Kim, W., Yoo, K.Y., et al. (2011) Grape Seed Extract Enhances Neurogenesis in the Hippocampal Dentate Gyrus in C57BL/6 Mice. Phytotherapy Research, 25, 668-674.

[23] Bachstetter, A.D., Jernberg, J., Schlunk, A., et al. (2010) Spirulina Promotes Stem Cell Genesis and Protects Against LPS Induced Declines in Neural Stem Cell Proliferation. PLOS ONE, 5, e10496. http://dx.doi.org/10.1371/journal.pone.0010496

[24] Nam, S.M., Yoo, D.Y., Kim, W., et al. (2011) Effects of S-Allyl-L-Cysteine on Cell Proliferation and Neuroblast Differentiation in the Mouse Dentate Gyrus. Journal of Veterinary Medical Science, 73, 1071-1075. http://dx.doi.org/10.1292/jvms.10-0557

[25] Pan, M., Li, Z., Yeung, V. and Xu, R.J. (2010) Dietary Supplementation of Soy Germ Phytoestrogens or Estradiol Improves Spatial Memory Performance and Increases Gene Expression of BDNF, TrkB Receptor and Synaptic Factors in Ovariectomized Rats. Nutrition \& Metabolism (Lond), 7, 75.

[26] Wang, Y., Li, M., Xu, X., et al. (2012) Green Tea Epigallocatechin-3-Gallate (EGCG) Promotes Neural Progenitor CeIl Proliferation and Sonic Hedgehog Pathway Activation during Adult Hippocampal Neurogenesis. Molecular Nutrition \& Food Research, 56, 1292-1303. Http://Dx.Doi.Org/10.1002/Mnfr.201200035

[27] Dias, G., Cavegn, N., Nix, A., et al. (2012) The Role of Dietary Polyphenols on Adult Hippocampal Neurogenesis: Molecular Mechanisms and Behavioural Effects on Depression and Anxiety. Oxidative Medicine and Cellular Longevity, 2012, Article ID: 541971. http://dx.doi.org/10.1155/2012/541971

[28] Kim, I.Y., Hwang, I.K., Choi, J.W., et al. (2009) Effects of High Cholesterol Diet on Newly Generated Cells in the Dentate Gyrus of C57BL/6N and C3H/HeN Mice. Journal of Veterinary Medical Science, 71, 753-758. 
http://dx.doi.org/10.1292/jvms.71.753

[29] Can, Ö.D., Ulupinar, E., Özkay, Ü.D., Yegin, B. and Öztürk, Y. (2012) The Effect of Simvastatin Treatment on Behavioral Parameters, Cognitive Performance, and Hippocampal Morphology in Rats Fed a Standard or a High-Fat Diet. Behavioural Pharmacology, 23, 582-592. http://dx.doi.org/10.1097/FBP.0b013e328356c3f2

[30] Inanami, O., Asanuma, T., Inukai, N., et al. (1995) The Suppression of Age-Related Accumulation of Lipid Peroxides in Rat brain by Administration of Rooibos Tea (Aspalathus linearis). Neuroscience Letters, 196, 85-88. http://dx.doi.org/10.1016/0304-3940(95)11853-O

[31] Vetulani, J. (2001) Drug Addiction. Part II. Neurobiology of Addiction. Polish Journal of Pharmacology, 53, $303-317$.

[32] Blum, K., Liu, Y., Shriner, R. and Gold, M.S. (2011) Reward Circuitry Dopaminergic Activation Regulates Food and Drug Craving Behavior. Current Pharmaceutical Design, 17, 1158-1167. http://dx.doi.org/10.2174/138161211795656819

[33] McCarthy, D.M., Brown, A.N. and Bhide, P.G. (2012) Regulation of BDNF Expression by Cocaine. Yale Journal of Biology and Medicine, 85, 437-446.

[34] Gardner, E.L. (2011) Addiction and Brain Reward and Anti-Reward Pathways. Advances in Psychosomatic Medicine, 30, 22-60. http://dx.doi.org/10.1159/000324065

[35] Orford, J. (2001) Addiction as Excessive Appetite. Addiction, 96, 15-31. http://dx.doi.org/10.1046/j.1360-0443.2001.961152.x

[36] Sussman, S. and Sussman, A.N. (2001) Considering the Definition of Addiction. International Journal of Environmental Research and Public Health, 8, 4025-4038.

[37] Yamamoto, Y., Tanahashi, T., Kawai, T., et al. (2009) Changes in Behavior and Gene Expression Induced by Caloric Restriction in C57BL/6 Mice. Physiological Genomics, 39, 227-235. http://dx.doi.org/10.1152/physiolgenomics.00082.2009

[38] Fang, J., Gu, J.W., Yang, W.T., Qin, X.Y. and Hu, Y.H. (2012) Clinical Observation of Physiological and Psychological Reactions to Electric Stimulation of the Amygdaloid Nucleus and the Nucleus Accumbens in Heroin Addicts after Detoxification. Chinese Medical Journal (English Edition), 125, 63-66.

[39] Carr, K.D. (2002) Augmentation of Drug Reward by Chronic Food Restriction: Behavioral Evidence and Underlying Mechanisms. Physiology \& Behavior, 76, 353-364. http://dx.doi.org/10.1016/S0031-9384(02)00759-X

[40] Ozburn, A.R., Harris, R.A. and Blednov, Y.A. (2008) Wheel Running, Voluntary Ethanol Consumption, and Hedonic Substitution. Alcohol, 42, 417-424. http://dx.doi.org/10.1016/j.alcohol.2008.04.006

[41] Varady, K.A. (2011) Intermittent Versus Daily Calorie Restriction: Which Diet Regimen Is More Effective for Weight Loss? Obesity Reviews, 12, e593-e601. http://dx.doi.org/10.1111/j.1467-789X.2011.00873.x

[42] Rothwell, P.E., Thomas, M.J. and Gewirtz, J.C. (2009) Distinct Profiles of Anxiety and Dysphoria during Spontaneous Withdrawal from Acute Morphine Exposure. Neuropsychopharmacology, 34, 2285-2295. http://dx.doi.org/10.1038/npp.2009.56

[43] Chen, K.W., Berger, C.C., Manheimer, E., et al. (2012) Meditative Therapies for Reducing Anxiety: A Systematic Review and Meta-Analysis of Randomized Controlled Trials. Depression and Anxiety, 29, 545-562. http://dx.doi.org/10.1002/da.21964

[44] Amen-Ra, N. (2011) Mind, Matter, Mathematics, \& Mortality: Meditations on a Momentous Metaphysical Theory. Amenta Press, Damascus.

[45] Bowen, S., Chawla, N., Collins, S.E., et al. (2009) Mindfulness-Based Relapse Prevention for Substance Use Disorders: A Pilot Efficacy Trial. Substance Abuse, 30, 295-305. http://dx.doi.org/10.1080/08897070903250084

[46] Jacobi, H. (1895) Jaina Sutras (Part II). In: Muller, F.M., Ed., The Sacred Books of the East, Oxford University Press, Oxford, 55. 